\title{
Électricité et Internet : le paradigme de l'iceberg
}

\author{
Bernard Aebischer ${ }^{1}$ et Jacques Roturier ${ }^{2}$ \\ 1. Centre for Energy Policy and Economics (CEPE), ETH-Zurich, baebischer@ethz.ch \\ 2. Professeur des Universités (e.r.), jacmon.roturier@laposte.net
}

\begin{abstract}
De l'électronique à l'informatique, puis au Web, nul ne s'étonne plus des possibilités extraordinaires dont tout un chacun apprécie les bénéfices. L'Internet serait-il à ce point exceptionnel que son coût global, et pas seulement financier, soit négligeable? Entreprise depuis presque 20 ans, mais longtemps occultée, l'étude de son impact énergétique montre que ce n'est pas le cas, ni au niveau du poste de travail ou de l'équipement ni surtout, à l'instar de l'iceberg, à celui de la part invisible des infrastructures ! Étayée ici par quelques données, cette évidence ne manque pas d'interroger ceux qui souhaiteraient que la passerelle numérique contribue à réduire la fracture Nord-Sud!
\end{abstract}

Quiconque prête attention à l'évolution respective de la physique et de l'informatique, depuis quatre ou cinq décennies, constatera les bénéfices mutuels de leur fertilisation croisée. Qu'il s'agisse des équipements scientifiques pour la première, des systèmes pour la seconde, il suffit ici de penser à ce qu'ont apporté, à l'une et à l'autre, les différents types de circuits électroniques depuis le transistor, à la fin des années 40 , jusqu'aux composants actuels ${ }^{1}$. Et, si le paradigme de Moore $^{2}$ demeure constamment avéré depuis 40 ans, nul ne doute que cela résulte des bénéfices que l'industrie électronique a su tirer de l'évolution de la physique des solides autant que de celle des lasers (pour ne citer qu'elles deux).

Perçue par tous comme absolument remarquable, une telle fertilisation est-elle pour autant sans défauts ? Accessible à tous? Exempte de tout risque environnemental ? Alors que la communauté des physiciens a déjà su discuter de l'impact de ses découvertes sur l'ensemble de la société, ce thème est, quant à lui, rarement débattu ! Bien entendu, notre communauté qui s'appuye sur l'électronique et l'informatique, a aussi contribué à l'éclosion du Web (inventé au $C E R N^{3}$ ), et ne saurait remettre en cause le « miracle » de l'Internet. Peut-elle, pour autant, ignorer que la "Société de l'Information » $(\mathrm{SI}){ }^{4}$ ne peut se construire sans une analyse exhaustive, c'est-àdire planétaire, de son coût global ${ }^{5}$ ce que, par exemple, Kofi A. Annan, Secrétaire Général des Nations Unies, soulignait ainsi ${ }^{6}$ : «...A Aujourd'hui, nombreux sont celles et ceux qui ne pourraient concevoir leur vie quotidienne sans les Technologies de l'Information et de la Communication TIC, sans cesse plus sophistiquées, qu'il s'agisse de la télévision, de la radio, du téléphone portable ou d'Internet. Et pourtant, dans les pays les plus pauvres du monde, des millions de personnes subissent les répercussions d'une "fracture numérique" qui les prive des avantages que présentent les TIC... ".

Bien entendu, ce « défi numérique » a fait l'objet de bien des débats en ce début du XXI ${ }^{\mathrm{e}}$ siècle lors des deux Sommets Mondiaux, organisés à l'initiative de l'ONU sur le continent africain, ce qui n'est d'ailleurs pas une coïncidence. Ainsi après Johannesburg (2002), celui de la Société de l'Information (SMSI/WSIS) analysant l'omniprésence de I'Internet lui assigne un objectif planétaire : raccorder à I'Internet la moitié de la population en 2015. Gigantesque est ce double défi de l'Internet « durable » et complexe l'analyse !

1. Une chronique raconte la part prise par des chercheurs français et allemands à l'histoire des inventions du transistor et du PC (http://www.avandormael.net/) 2. Doublement tous les 18 mois de la densité d'intégration des composants au sein d'un circuit intégré (http://informatech.online.fr/articles/cpu/index.php).

3. How the Web was Born: The Story of the World Wide Web, Robert Cailliau, James Gillies (Oxford University Press, 2000) ISBN 0192862073

4 . Le concept « SI », vu ici comme une ère au même titre que l'ère industrielle,

caractérise les liens entre les TIC et la société contemporaine.

5 . Cette notion recouvre l'ensemble des coûts (investissement, fonctionnement et fin de vie) de tout équipement.

6. Adresse aux participants à la Journée Mondiale des Télécommunications 2004.

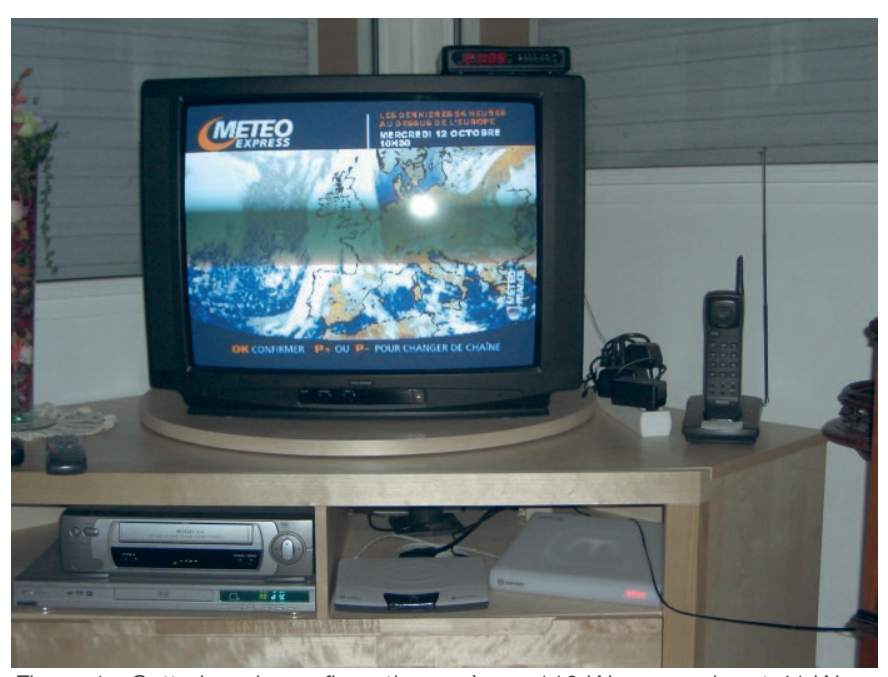

Figure 1 : Cette banale configuration "pèse " $110 \mathrm{~W}$ en marche et $41 \mathrm{~W}$ en veille. En mode Internet, les $65 \mathrm{~W}$ du téléviseur entraînent un appel quasiéquivalent de puissance supplémentaire (niveau local et niveau des infrastructures).

À un modeste niveau, nous en présentons ici l'une des facettes, certes restreinte, une tâche à laquelle nous nous consacrons depuis presque 20 ans : l'impact énergétique et environnemental des TIC, constituant l'ossature même de la SI. Quelques étapes jalonnant la prise en compte énergétique de ces technologies étant d'abord succinctement présentées, nous résumerons ensuite des données, issues entre autres de travaux menés avec des fournisseurs de services, conduisant à des ordres de grandeur du «poids » des infrastructures.

\section{L'électricité des TIC est-elle négligeable?}

Logiquement issu de l'invention du microprocesseur, et né avec le premier choc pétrolier (1973), le PC a très vite contribué aux économies d'énergie. Et aussi largement utilisé, comme en témoigne la constante progression de ses applications : micro-informatique, bureautique, domotique, télématique... des mots devenus obsolescents ! Autres temps, autres modes avec les Web, Wifi/AsFi ${ }^{7}$ et autres Bluetooth! Derrière tout ceci beaucoup de technologies certes, mais aussi, ce qui est moins connu, de plus en plus d'électricité ! Nos premières analyses l'ont démontré dès la fin des années 80 et, acquise d'abord indépendamment au sein de nos institutions, notre expérience conjointe a été souvent mise en commun depuis lors [1]. Sans entrer dans trop de détails, nous citerons à ce sujet quelques faits ou évidences dont nos laboratoires furent parmi les premiers à souligner l'importance : I'Université de Bordeaux 1 à partir de 1987, en colla-

7. WiFi/AsFi : Wireless Fidelity/ Accès sans Fil à l'internet. 
boration avec le LBNL-UC Berkeley et le MIT d'une part [2] et, d'autre part, ETH-Zurich, acteur essentiel de la politique énergétique suisse [3].

Nous avons ainsi contribué, avec nos collègues de US-EPA (Agence Fédérale de Protection de l'Environnement), à la définition du label «Energy Star® » en 1992. La Commission Européenne créait alors les groupes de travail OT3E et Macebur [4], associant chercheurs et industriels pour évaluer l'impact énergétique des TIC et proposer des mesures permettant d'exploiter le gisement d'économies réalisable. La plus marquante de celles-ci a été le début d'une (longue et difficile) négociation transatlantique améliorant le programme Energy Star® et l'appliquant à l'ensemble du territoire de l'UE à partir de 2001. Ces initiatives n'ont cependant pu freiner la croissance de l'impact électrique des TIC au cours des 15 dernières années à cause de la croissance du parc, mais aussi de celle de la durée d'utilisation devenant quasi-continue, alors que la possibilité de mettre en veille ${ }^{8}$ les équipements demeurait souvent lettre morte. Ceci peut expliquer pourquoi, dans les établissements d'enseignement supérieur, la demande d'électricité augmente à un rythme souvent bien plus élevé que dans l'ensemble du pays.

Par ailleurs, dans le secteur résidentiel, les équipements électroménagers et de loisirs de nouvelle génération y posent des problèmes de gestion de la consommation d'électricité en termes semblables à ceux des PC, y compris leur incontournable tendance à la mise en réseau. L'AIE/OCDE a ainsi ciblé les TIC comme l'un des axes majeurs d'une politique de Maîtrise de la Demande d'Électricité (MDE) ${ }^{9}$. De fait, la banalisation de l'Internet, support même de la SI, impose la dissémination systématique des technologies numériques dans la quasi-totalité des équipements électriques et ce, dans tous les secteurs d'activités. En sus d'un coût énergétique élevé, élément essentiel du point de vue de la durabilité, le concept TIC « durable » doit aussi être complété par un autre aspect. "Durable » étant pris ici au sens, non des bénéfices qui résultent de l'usage des TIC, mais de leur coût global, rappelons ici, à titre indicatif, ce qu'est l'empreinte écologique imaginée par W. Rees et M. Wackernagel, au début des années 90. Basée sur la notion de biocapacité de la planète, une donnée semi-quantitative à manipuler avec précaution, elle attribue à chaque Terrien une ressource égale à ce que fournit, pour tout

\begin{tabular}{|l|r|r|r|r|r|}
\hline & $\begin{array}{c}2001 \\
\text { TWh/an }\end{array}$ & $\begin{array}{r}2010 \\
\text { TWh/an }\end{array}$ & $\begin{array}{c}2001 \\
\%\end{array}$ & $\begin{array}{c}2010 \\
\%\end{array}$ & $\begin{array}{c}2001- \\
2010 \\
\% / \mathrm{an}\end{array}$ \\
\hline $\begin{array}{l}\text { Secteur } \\
\text { résidentiel }\end{array}$ & 19,1 & 24,5 & 50 & 44 & $+2,8$ \\
\hline $\begin{array}{l}\text { Secteur } \\
\text { tertiaire }\end{array}$ & 7,8 & 7,6 & 21 & 14 & $-0,2$ \\
\hline $\begin{array}{l}\text { Infrastructures } \\
\text { résidentiel }\end{array}$ & 3,4 & 6,4 & 9 & 12 & $+7,3$ \\
\hline $\begin{array}{l}\text { Infrastructures } \\
\text { tertiaire }\end{array}$ & 5,4 & 11,1 & 14 & 20 & $+8,3$ \\
\hline $\begin{array}{l}\text { Infrastructures } \\
\text { publiques }\end{array}$ & 2,3 & 5,8 & 6 & 10 & $+11,1$ \\
\hline \multicolumn{1}{|c|}{ Total } & 38,0 & 55,4 & 100 & 100 & $+4,3$ \\
\hline
\end{tabular}

Tableau 1 : Consommation d'électricité 2001 et 2010 en Allemagne des équipements et installations de TIC (d'après Cremer et al. [9]).

8. Trois sites à consulter à ce sujet

http://energyefficiency.jrc.cec.eu.int/energystar/index.htm concernant Energy Star en Europe, celui du Group for Efficient-Energy Appliances http://www.gealabel.org/criteria.htm et enfin celui de IEEE pour la nouvelle norme IEEE 1621-2004 (IEEE Standard for User Interface Elements in Power norme IEEE 1621-2004 (IEEE Standard for User Interface Elements in Power
Control of Electronic Devices Employed in Office/Consumer Environments) http://standards.ieee.org/ (contact Bruce Nordman, via BNordman@LBL.gov ). 9.Things That Go Blip in the Night (OECD-IEA Publications 2001). ce qui est nécessaire à la vie et à l'élimination des déchets produits, une fraction de l'espace terrestre et maritime égale pour tous soit, en gros, 2 hectares. Selon une étude de Frey et al. [5], effectuée à Brunel University (UK), l'empreinte écologique d'un seul PC atteint $9 \%$ de la biocapacité par habitant, une valeur plus que significative. Soyons donc lucides ! Dans l'Euro 25 de 2006, si la puissance électrique requise par les TIC n'a pu qu'augmenter continûment depuis notre évaluation de 1994 (10 GW), le gisement d'économies $(\approx 50 \%)$ demeure virtuel... et leur impact environnemental de moins en moins négligeable.

\section{Et celle des infrastructures?}

Ce n'est que récemment, à l'occasion du SMSI, que leur impact énergétique a été analysé. Les bilans sont donc encore fragmentaires, mais il nous semble utile de présenter quelques-unes des informations disponibles. Avant de finir par un point de vue macroéconomique, nous examinerons des données issues d'études de fournisseurs de services. Prenons l'exemple de la téléphonie mobile; bien qu'une analyse comparative de l'impact électrique soit malaisée, nous disposons de quelques éléments [1]. On sait ainsi que si un terminal GSM de 1996 consomme 4 à 5 fois plus qu'un modèle 2001, la part électricité des infrastructures évolue beaucoup plus lentement : pour les mobiles de 1 ère génération, la répartition relative de la consommation annuelle entre un terminal GSM et son infrastructure a été évaluée à $0,55 \mathrm{kWh}$ et 7,62 kWh soit $7 \%$ et $93 \%$ respectivement [6]. Avec la technologie UMTS, une telle répartition relative, qui dépend d'une multitude de facteurs (taux d'utilisation de l'infrastructure, performance énergétique des terminaux....), peut varier dans un sens ou dans l'autre. En téléphonie mobile, il est cependant établi que la part de la demande d'électricité de l'infrastructure sera de $50 \%$ au moins.

Sur un plan plus global, rappelons une étude demandée par le US-DOE, en réponse à un début de polémique sur l'impact de I'Internet. Selon son auteur, Kurt Roth [7, 8], PC et écrans sont en tête des usages, avec environ $40 \%$ du total des TIC. Les infrastructures, avec serveurs, réseaux informatiques et téléphoniques, onduleurs, mais hors climatiseurs, qui sont deuxièmes (environ 30\%), verront leur part relative augmenter à brève échéance. Aux USA, la consommation d'électricité des TIC stricto sensu est ainsi de l'ordre de $3 \%$ du total de la consommation nationale. Quant à celle des circuits électroniques et autres processeurs « invisibles " mais omniprésents dans les véhicules, les bâtiments, les machines industrielles, si elle n'y fait pas encore l'objet d'une estimation, il ne fait aucun doute que cette part continuera à croître. Dans les pays industrialisés européens, où la consommation par habitant est trois fois moins élevée en moyenne qu'aux USA, les TIC pèsent de $7 \%$ (Tableau 1) en Allemagne [9], où la consommation d'électricité a été de 569 TWh en 2003, à $10 \%$ environ en Suisse [10]. Des chiffres, dont la figure 2 montre qu'ils dépassent la consommation totale d'électricité per capita dans beaucoup de pays du Sud!

\section{Conclusion}

Les quelques observations faites ici risquent donc de heurter bien des idées reçues. À court terme pourtant, dans les pays de l'OCDE du moins, il est vraisemblable que les TIC et leurs applications deviendront ainsi l'un des plus importants secteurs de consommation d'électricité. Quant à leur impact environnemental, à peine esquissé ici, il ne peut plus être négligé. II ne fait donc aucun doute que leur « durabilité » ne saurait être améliorée sans des actions volontaristes... en matière de sensibilisation des scientifiques eux-mêmes, ainsi que d'information et de formation de tous les usagers ! Rappelons ainsi le succès médiatique de la proposition faite par des chercheurs du MIT au SMSI-Tunis de diffuser partout 


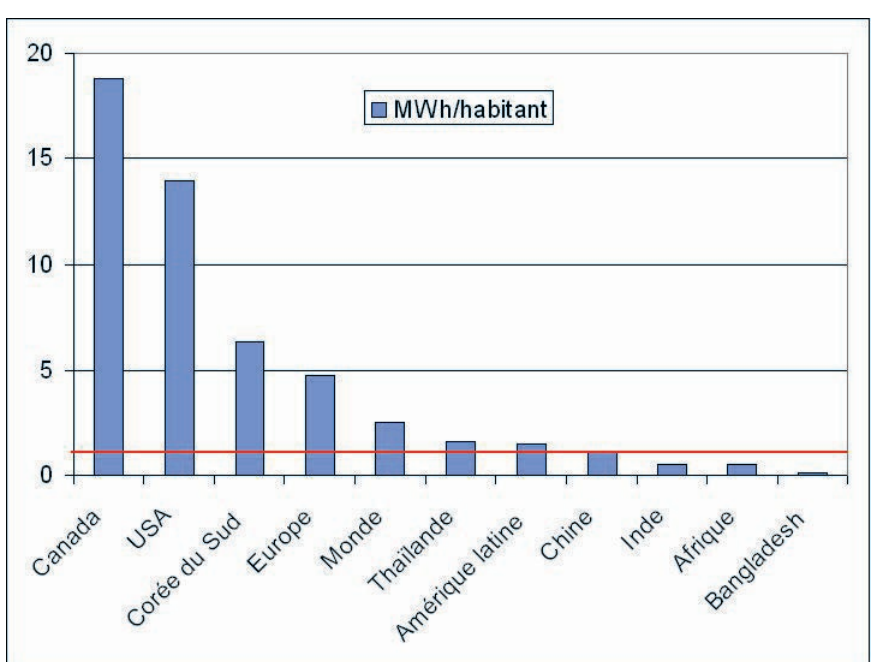

Figure 2 : Consommation totale annuelle d'électricité par habitant dans différentes régions du monde (barres) et de celle des TIC en Suisse et aux USA (ligne rouge) (d'après Aebischer [10]).

dans le monde leur PC à 100 \$. Supposant résolues les questions de maintenance, cette technologie serait-elle le remèdemiracle assurant un véritable "worldwide" à l'Internet ? Nous n'en sommes pas persuadés, tant les infrastructures dans nombre de parties du globe nécessitent des investissements très loin d'être encore décidés. Ayant le privilège sans limites apparentes aujourd'hui, de pouvoir développer et adapter les incontournables réseaux électriques et de télécommunications, il se pourrait donc que les pays du Nord accèdent seuls, et pour longtemps, à la Société de l'Information. Dans les pays du Sud, où l'existence et la fiabilité des réseaux existants sont aujourd'hui des soucis quotidiens, assurer un accès normal au Web est donc un incontestable défi qui, à l'évidence, ne sera pas relevé par les seuls physiciens bien que, nous semble-t-il, beaucoup d'entre eux aient vocation à y contribuer.

\section{Références}

[1] Aebischer B. et Roturier J., "Infrastructures de la Société de I'Information : un gigantesque défi énergétique ", Contribution des auteurs à Infrastructures et énergie (2006) à paraitre dans la collection "Énergie, Environnement et Société" du CUEPE, Université de Genève. http://www.unige.ch/cuepe/html/biblio/biblio_u.php?mjc=128

[2] Norford L., Rabl A., Harris J.P., Roturier J., "Electronic Office Equipment: The Impact of Market Trends and Technology" in ELECTRICITY : Efficient End-Use and New Generation Technologies and Their Planning Implications ed. by T.B. Johansson, B. Bodlund, R.M. Williams, Lund University Press (1989).

[3] Spreng D. "Computer as Energy Consumers", Energy Policy, Vol. 19, №. 7, Sept. 1991.

[4] Roturier J. et al., Rapport final (1994) du Groupe de Travail OT3E : "Energy-Efficient Office Technologies in Europe" (Contrat CEDGXVII - Université Bordeaux 1 \# XVII/4.1301/E/92-07) et Rapport final (1998) du Groupe de Travail MACEBUR: "Energy-Efficient Information and Communication Technologies in Europe : The 1 W/1 VA Challenge" (Contrat CE-DGXVII - IN2P3 \# XVII/4.1301/S/94-87).

[5] Frey S.D., Harrison D.J., Billett E.H., "Environmental assessment of electronic products using LCA and ecological footprint" - Joint International Congress and Exhibition. Electronics goes green 2000, Berlin, Allemagne.

[6] Souchon L., Mémoire de Master SIPT (2005).

[7] Roth K. et al., "Energy Consumption by Office and Telecommunications Equipment in Commercial Buildings", Volume I : Energy Consumption Baseline, ADL, Cambridge, MA, USA, 2002. http://www.tiaxllc.com/aboutus/pdfs/officeequipvol1.pdf

Volume II : Energy Savings Potential, TIAX LLC, Cambridge, MA, USA, 2004.

http://www.tiaxllc.com/aboutus/pdfs/DOE_Energy_Consumption_120 4_Rpt_033105.pdf
[8] Roth K., 2005 "The Potential to Reduce Information and Communication Technology Energy Consumption", CEPEColloquium, ETHZ, Zürich, Oct. 2005.

http://www.cepe.ch/download/news-events/ICT_Savings_CEPE2005.pdf

[9] Cremer C., Aebischer B. et al., "Energy Consumption of Information and Communication Technology in Germany up to 2010, 2003".

http://www.cepe.ch/download/projects/INFO-KOM/ISI\%2BCEPE-ICTenglish.pdf

[10] Aebischer B., 2002 " Informationstechnologie : Energiesparer oder Energiefresser? " EMPA-Akademie Wissenschaftsapéro 28. 10. 2002. 